\title{
In Vivo Assessment of Parenteral Formulations of Oligo(3-Hydroxybutyric Acid) Conjugates with the Model Compound Ibuprofen
}

\author{
Pawel Stasiak, ${ }^{1}$ Malgorzata Sznitowska, ${ }^{1,5}$ Carsten Ehrhardt, ${ }^{2}$ Maria Luczyk-Juzwa, ${ }^{3}$ and Pawel Grieb ${ }^{4}$
}

\begin{abstract}
Received 7 May 2010; accepted 9 November 2010; published online 19 November 2010
Abstract. Polymer-drug conjugates have gained significant attention as pro-drugs releasing an active substance as a result of enzymatic hydrolysis in physiological environment. In this study, a conjugate of 3hydroxybutyric acid oligomers with a carboxylic acid group-bearing model drug (ibuprofen) was evaluated in vivo as a potential pro-drug for parenteral administration. Two different formulations, an oily solution and an o/w emulsion were prepared and administered intramuscularly (IM) to rabbits in a dose corresponding to $40 \mathrm{mg}$ of ibuprofen/kilogramme. The concentration of ibuprofen in blood plasma was analysed by HPLC, following solid-phase extraction and using indometacin as internal standard (detection limit, $0.05 \mu \mathrm{g} / \mathrm{ml}$ ). No significant differences in the pharmacokinetic parameters $\left(C_{\max }, T_{\max }\right.$, AUC) were observed between the two tested formulations of the 3-hydroxybutyric acid conjugate. In comparison to the non-conjugated drug in oily solution, the relative bioavailability of ibuprofen conjugates from oily solution, and o/w emulsion was reduced to $17 \%$ and $10 \%$, respectively. The 3 hydroxybutyric acid formulations released the active substance over a significantly extended period of time with ibuprofen still being detectable $24 \mathrm{~h}$ post-injection, whereas the free compound was almost completely eliminated as early as $6 \mathrm{~h}$ after administration. The conjugates remained in a muscle tissue for a prolonged time and can hence be considered as sustained release systems for carboxylic acid derivatives.
\end{abstract}

KEY WORDS: drug conjugates; ibuprofen; oligo(3-hydroxybutyrates); pharmacokinetics; pro-drugs.

Along with aqueous suspensions, oily solutions, microspheres and implants, conjugates of drug substances with biocompatible and biodegradable polymers have been widely investigated for drug delivery applications $(1,2)$. In contrast to technologies based on drug incorporation into the polymer matrix, conjugation to polymers significantly changes not only the pharmacokinetic properties of the drug, but also its chemical and physicochemical characteristics, including, e.g., solubility, stability and reactivity. Some of these conjugates, mainly with polyethylene glycols (PEG), have been approved and introduced into the clinic as safe and effective alternative to the parent drugs (1). Alternatives to PEG include polysaccharides (e.g., dextrans), vinyl polymers, poly amino acids and dendrimers (3). Current research in the field of biopolymers and biomaterials is focused on the development of novel

\footnotetext{
${ }^{1}$ Department of Pharmaceutical Technology, Medical University of Gdansk, ul. Hallera 107, 80-416, Gdansk, Poland.

${ }^{2}$ School of Pharmacy and Pharmaceutical Sciences, Trinity College Dublin, Dublin, Ireland.

${ }^{3}$ Centre of Polymer and Carbon Materials, Polish Academy of Sciences, Zabrze, Poland.

${ }^{4}$ Mossakowski Medical Research Centre, Polish Academy of Sciences, Warsaw, Poland.

${ }^{5}$ To whom correspondence should be addressed. (e-mail: msznito@ gumed.edu.pl)
}

polymers which are characterised by well-established metabolism route, demonstrate absence of toxicity and improve the pharmacokinetic properties of drugs, mainly by extending their half-life time, enhancing their solubility, reducing immunological reactions as well as fast elimination by enzymatic degradation (4).

A novel approach for drug conjugation are oligomers of 3-hydroxybutyric acid (OHB) (5) which are synthesised by a number of microorganisms and can also be found in animal cells (6). Conjugates of carboxylic acid group-bearing non-steroidal anti-inflammatory drugs (NSAIDs) with OHB have recently been developed and characterised at the Centre of Polymer and Carbon Materials of Polish Academy of Sciences. It was reported that these conjugates are biocompatible and show high stability in aqueous solutions of pH 6.0-8.0 (7-9). Ibuprofen-OHB (Fig. 1) is a semi-solid substance with good solubility in organic solvents including oil (vegetable oils and Miglyol). It also marked resistance against hydrolytic enzymes (e.g., pancreatin) (9), however, no data on the conjugate's pharmacokinetics following parenteral administration has been available.

In the present study we discuss the in vivo pharmacokinetics of ibuprofen-OHB after IM injection into rabbits from two different formulations, an oily solution and an o/w emulsion. We also report on the development of a high performance liquid chromatography (HPLC) method, preceded by SPE that was developed to analyse ibuprofen concentrations in the collected plasma samples. 


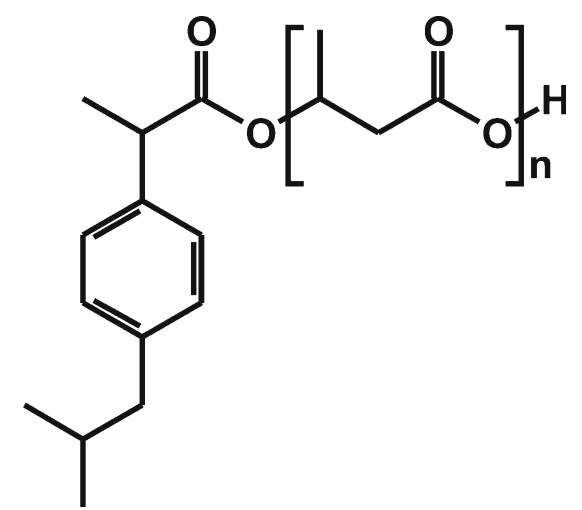

Fig. 1. Chemical structure of the conjugate of ibuprofen with oligomers of 3-hydroxybutyric acid $(n=3-7)$

\section{MATERIALS AND METHODS}

\section{Chemicals}

Racemic ibuprofen (IB, Shasun, Chennai, India) was kindly donated by Polfa Kutno (Kutno, Poland) and indometacin (IND) was a gift from Jelfa (Jelenia Gora, Poland). Ibuprofen-OHB (M.W. $570 \mathrm{Da}$ ) containing approximately $2.5 \%(w / w)$ of non-conjugated IB was provided by the Centre of Polymer and Carbon Materials (Zabrze, Poland). Synthesis and analytical methods confirming purity, structure and molecular weight of the conjugate have been described elsewhere $(9,10)$.

Acetonitrile (Merck, Darmstadt, Germany) and methanol (POCh, Gliwice, Poland) were of HPLC grade. Reagents for preparation of buffered solutions were also purchased from POCh. For drug formulations, Miglyol 812 (medium-chain triglycerides, Caesar and Loretz, Hilden, Germany), 86\% (w/w) glycerol (Laboratorium Galenowe, Olsztyn, Poland) and Tween 80 (Fluka, Buchs, Switzerland) were used.

\section{Instrumentation and Chromatographic Conditions}

The HPLC system (Merck Hitachi, Darmstadt, Germany) consisted of an isocratic HPLC pump (L-7100), autosampler (L7200), UV-vis detector (L-7420) and integrator (D-7000). As stationary phase a LiChrospher 100 RP-18 column $(5 \mu \mathrm{m}$, Merck, Darmstadt, Germany) was used and the mobile phase was a mixture of $10 \mathrm{mM}$ phosphate buffer $(\mathrm{pH} 7.0)$ and acetonitrile $70: 30(v / v)$. Flow rate was set to $1 \mathrm{ml} / \mathrm{min}$, and detection was performed at $220 \mathrm{~nm}$.

\section{Standard Solutions and Sample Preparation}

For calibration, solutions of IB $(5,12.5,25,50,125,500$ and $1,250 \mu \mathrm{g} / \mathrm{ml}$ ) and a solution of an internal standard IND $(25 \mu \mathrm{g} / \mathrm{ml})$ in methanol were used. Twenty microlitres of IB solution and $20 \mu \mathrm{l}$ of the internal standard solution were added to $500 \mu \mathrm{l}$ of blood plasma and vortexed for $10 \mathrm{~s}$. Then, $100 \mu \mathrm{l}$ of a $2 \%(w / w)$ solution of phosphoric acid and $600 \mu \mathrm{l}$ of $100 \mathrm{mM}$ phosphate buffer ( $\mathrm{pH}$ 6.0) were added, and the sample was vortexed again for $30 \mathrm{~s}$. SPE columns (Strata-X $33 \mu \mathrm{m}$, Phenomenex, Torrance, USA) were initially washed with $1 \mathrm{ml}$ of methanol followed by $1 \mathrm{ml}$ of water according to manufacturer's directions (11). The samples were applied to the SPE columns and allowed to pass through without vacuum being applied. The columns were then washed with $700 \mu \mathrm{l}$ of $5 \%(v / v)$ methanol in water. A vacuum was applied for $1 \mathrm{~min}$ to remove residual solvents. The elution was carried out using $1 \mathrm{ml}$ of methanol. Collected solutions were centrifuged $(3,000 \times g)$ and $950 \mu \mathrm{l}$ were transferred to HPLC vials and the solvent was allowed to evaporate at $37^{\circ} \mathrm{C}$ under a gentle stream of air. The residue was then dissolved in $200 \mu \mathrm{l}$ of methanol, and $20 \mu \mathrm{l}$ were injected into the HPLC system.

\section{Validation}

Specificity of the method was determined at $220 \mathrm{~nm}$ by comparing the chromatograms after extraction of blank plasma and plasma spiked with IB $(1 \mu \mathrm{g} / \mathrm{ml})$ and IND $(1 \mu \mathrm{g} / \mathrm{ml})$. Linearity was tested by extraction and injection of nine sets of IB standard solutions in the concentration range $0.2-50 \mu \mathrm{g} / \mathrm{ml}$. Precision was calculated at different concentrations-0.2, 5 and $20 \mu \mathrm{g} / \mathrm{ml}$. Repeatability and reproducibility were calculated from the chromatograms of the same samples injected at different time points during one day $(n=3)$ or on three different days, respectively. Accuracy was calculated by applying the test procedure four times for three different IB concentrations $(0.5$, 2 and $5 \mu \mathrm{g} / \mathrm{ml}$ ). Recovery rates were determined by comparing extracted spiked plasma samples with non-extracted solutions of standards in methanol (IB 1.25, 5 and $12.5 \mu \mathrm{g} / \mathrm{ml}$ ). Limits of detection and quantification were also determined.

\section{Drug Formulations}

For parenteral administration of ibuprofen-OHB, a $25 \%(w / w)$ oily solution in Miglyol 812 and a $10 \%$ o/w emulsion ( $20 \%$ of oily phase) were prepared. The oily solution was obtained by dissolving ibuprofen-OHB in Miglyol at $60^{\circ} \mathrm{C}$, followed by sterilisation through filtration ( $0.2 \mu \mathrm{m}$ cellulose acetate filters). The emulsion containing ibuprofen-OHB, polysorbate $80(2 \% w / w)$, glycerol $(2.8 \%$ $w / w)$, Miglyol 812 and water was prepared by high-speed homogenisation with an Ultra-Turrax T-25 (Janke and Kunkel, Staufen, Germany). Sterilisation of the emulsion formulation was performed by autoclaving $\left(121^{\circ} \mathrm{C}, 15 \mathrm{~min}\right)$. An oily solution of non-conjugated IB $(5 \% w / w)$ in Miglyol 812 was prepared as control and also sterilised by filtration as described above. No adverse effects of the thermal sterilisation on the emulsion stability were observed (9). Parameters investigated were $\mathrm{pH}$, droplet size and ibuprofen-OHB concentration. All formulations were prepared at most 5 days prior to the start of the in vivo experiments, however stability of the formulations at $5 \pm 2{ }^{\circ} \mathrm{C}$ was investigated within 90 days after preparation (9).

\section{Pharmacokinetics of Ibuprofen after Ibuprofen-OHB Administration in Rabbits}

The animal experiments were designed to follow guidelines of the National Ethics Committee. The application was reviewed and approved by the 4th Local Ethics Committee in Warsaw (Permission $N^{\circ}$ 25/2007). Eighteen adult rabbits (less than 1 year old, nine males and nine females) with body 
weights of $2.73 \pm 0.32 \mathrm{~kg}$ were used. The animals were purchased from Hodowla Krolikow Rasowych (Wielichowo, Poland) and left to acclimatise for 1 week in the animal facility, prior to the start of the study. The animals were fed with standard rodent chow and had unrestricted access to fresh water. The study was supervised and assisted by a qualified veterinary surgeon and preceded accordingly to the application approved.

The animals were divided into three groups of six (males and females) and each group received one of the investigated formulations by injection into the musculus semimembranosus. Ibuprofen in oily solution was administered at doses of $25 \mathrm{mg} / \mathrm{kg}$ and ibuprofen-OHB in oil or in emulsion-at doses of $120 \mathrm{mg} / \mathrm{kg}$ (corresponding to $40 \mathrm{mg} / \mathrm{kg}$ of IB). The total injection volume, depending on the formulation, was between 1.1-3.5 ml. One-millilitre blood samples were collected in heparinised plastic tubes (Medlab Products, Raszyn, Poland) from the marginal ear vein prior to drug administration and at $1,3,6,12,24,36$, and $48 \mathrm{~h}$ after injection. Samples were centrifuged at room temperature $(15 \mathrm{~min}, 3,000 \times \mathrm{g})$ and the plasma was separated and stored at $-20^{\circ} \mathrm{C}$ until further analysis. After addition of $20 \mu \mathrm{l}$ of the internal standard (IND, $25 \mu \mathrm{g} / \mathrm{ml}$ ) to $500 \mu \mathrm{l}$ of the plasma, samples were acidified, diluted with $600 \mu$ phosphate buffer ( $\mathrm{pH}$ 6.0) and analysed as described above.

\section{Data Analysis}

The calibration curves were calculated by linear regression of the IB/IND peak area ratio versus IB concentration. The $\mathrm{AUC}_{0-24 \mathrm{~h}}$ (for IB formulation) or $\mathrm{AUC}_{0-48 \mathrm{~h}}$ (for ibuprofen-OHB formulations) was calculated using linear trapezoidal rules. Statistical analysis was performed using KruskalWallis one-way analysis of variance. Individual differences between the formulations were identified by Dunn's post hoc test or by Mann-Whitney rank sum test. A significance level of $p<0.05$ denoted significance in all cases.

Relative bioavailability (EBA) was calculated using Eq. (1).

$$
E B A=\frac{A U C_{\text {ibuprofen-OHB }} \times D_{I B}}{A U C_{I B} \times D_{\text {ibuprofen-OHB }}} \times 100 \%
$$

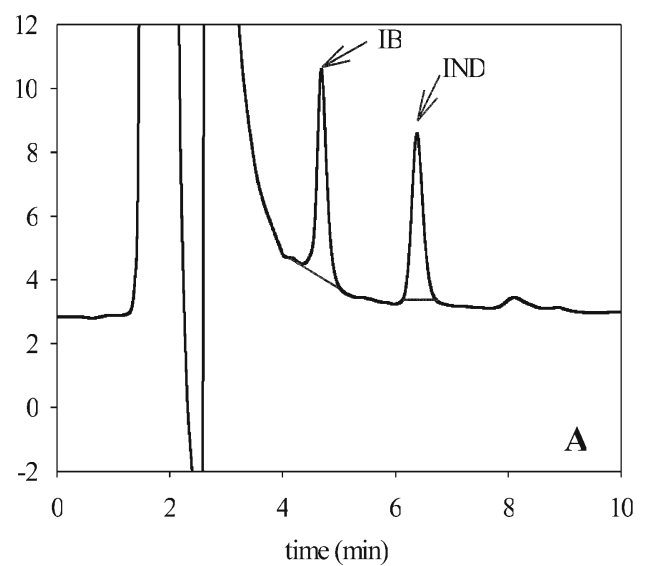

where AUC is area under curve and $D$ is a dose of ibuprofen-OHB or IB.

\section{RESULTS}

\section{Analytical Method}

The chromatographic conditions allowed for an evident separation of IB from the internal standard. Figure $2 \mathrm{a}$ represents a sample chromatogram of plasma collected during the experiment, spiked with IND and subjected to SPE. Ibuprofen and IND are represented by peaks with retention times at 4.5 and $6.3 \mathrm{~min}$, respectively. Blank plasma samples did not show any peaks with retention times after $4 \mathrm{~min}$ (Fig. 2b). This suggests absence of interferences of the analysed substances (IB and IND) with endogenous compounds. A linear correlation between IB/IND peak area ratio and IB concentration $\left(\mathrm{C}_{\mathrm{IB}} 0.2\right.$ $20 \mu \mathrm{g} / \mathrm{ml})$ was clearly demonstrated $\left(R^{2}=0.9999\right)$ and is given by Eq. (2).

$$
\mathrm{IB} / \mathrm{IND}=0.618 \times \mathrm{C}_{\mathrm{IB}}+0.024
$$

The coefficient of variation for the repeatability (withina-day) and the reproducibility (in-between-days) analyses was $2.06 \pm 3.18 \%$ and $10.05 \pm 7.03 \%$, respectively. The percentage of recovery from rabbit plasma was $75.5 \pm 8.3 \%$ for IB and $84.5 \pm 7.6 \%$ for IND. The method proved to be accurate, with $99.3 \pm 1.5 \%$ of IB detected in the analysed samples. The quantification and detection limits were 0.2 and $0.05 \mu \mathrm{g} / \mathrm{ml}$, respectively.

\section{Drug Formulations}

The properties and stability of ibuprofen-OHB drug formulations as well as physicochemical characteristics of the conjugate have been discussed elsewhere (9). Briefly, concentrations of ibuprofen-OHB, size of the emulsion oily droplets as well as $\mathrm{pH}$ did not change significantly during the stability test $(3 \%$ decrease in concentration after 90 days was observed). On average, upper limit of diameter for $50 \%$ of droplets $\left(\mathrm{d}_{0.5}\right)$ was approximately

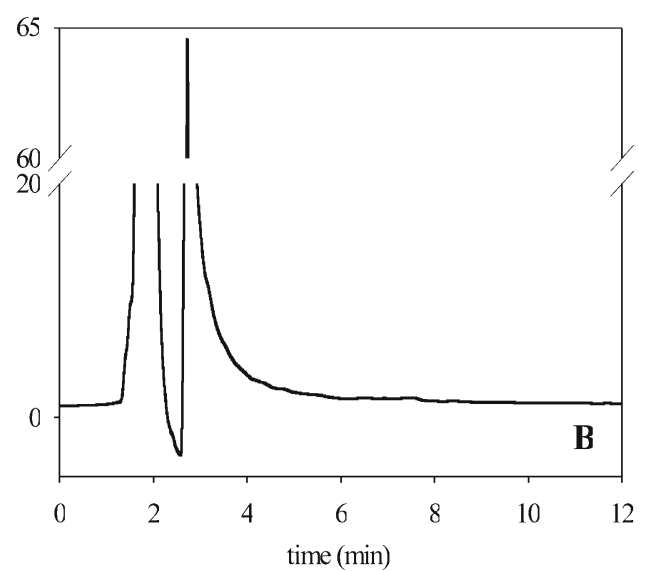

Fig. 2. Sample HLPC chromatogram of analysed plasma collected during the experiment, spiked with an internal standard (IND, $2.5 \mu \mathrm{g} / \mathrm{ml}$ ) and subjected to solid-phase extraction a and representative HPLC chromatogram of extracted blank plasma sample $\mathbf{b}$ 
Table I. Individual Plasma Concentration Profiles of IB Administered in a Form of Non-nonconjugated Substance and in a Form of the Conjugate (Ibuprofen-OHB) as an Oily Solution and o/w Emulsion.

\begin{tabular}{|c|c|c|c|c|c|c|c|c|}
\hline \multirow[b]{2}{*}{ Animal $N^{\circ}$} & \multicolumn{7}{|c|}{ Time (h) } & \multirow[b]{2}{*}{$\operatorname{AUC}_{0-\mathrm{t}}(\mu \mathrm{g} / \mathrm{ml} \cdot \mathrm{h})$} \\
\hline & 1 & 3 & 6 & 12 & 24 & 36 & 48 & \\
\hline \multicolumn{9}{|c|}{ Ibuprofen-OHB oily solution (40 mg IB/kg) } \\
\hline 1 & 1.24 & 0.58 & 0.45 & 0.23 & $0.71^{r}$ & 0.00 & 0.00 & \\
\hline 2 & 1.10 & 1.17 & 0.61 & $0.18^{a}$ & 0.25 & 0.00 & 0.00 & \\
\hline 3 & 0.82 & 0.55 & 0.46 & $0.15^{a}$ & $0.11^{a}$ & 0.00 & 0.00 & \\
\hline 4 & 0.87 & 0.79 & 0.60 & 0.67 & 0.29 & 0.00 & 0.00 & \\
\hline 5 & 1,68 & 1.61 & 1.00 & 0.28 & 0.20 & 0.36 & 0.25 & \\
\hline 6 & 1.86 & 1.38 & 0.60 & $0.15^{a}$ & $0.08^{a}$ & 0.00 & $0.02^{a}$ & \\
\hline $\operatorname{Mean} \pm$ SD & $1.26 \pm 0.42$ & $1.01 \pm 0.44$ & $0.62 \pm 0.20$ & $0.28 \pm 0.20$ & $0.19 \pm 0.09$ & $0.06 \pm 0.14$ & $0.04 \pm 0.1$ & $13.2 \pm 6.7$ \\
\hline \multicolumn{9}{|c|}{ Ibuprofen-OHB emulsion (40 mg IB/kg) } \\
\hline 7 & $0.28^{r}$ & $0.16^{r}$ & $0.20^{r}$ & $0.18^{a}$ & $0.03^{a}$ & 0.00 & $0.09^{a}$ & \\
\hline 8 & 0.91 & 0.97 & 0.75 & 0.31 & $0.03^{a}$ & 0.00 & 0.00 & \\
\hline 9 & 0.56 & 0.32 & 0.30 & $0.19^{a}$ & $0.04^{a}$ & 0.00 & 0.00 & \\
\hline 10 & 1.19 & 0.72 & 0.41 & $0.19^{a}$ & $0.02^{a}$ & 0.00 & 0.00 & \\
\hline 11 & 0.88 & 0.38 & 0.34 & $0.18^{a}$ & $0.06^{a}$ & 0.00 & $0.03^{a}$ & \\
\hline 12 & 1.58 & 1.33 & 0.63 & $0.13^{a}$ & $0.03^{a}$ & 0.00 & 0.00 & \\
\hline Mean \pm SD & $1.02 \pm 0.38$ & $0.74 \pm 0.42$ & $0.49 \pm 0.19$ & $0.20 \pm 0.06$ & $0.04 \pm 0.01$ & 0.00 & $0.02 \pm 0.04$ & $7.9 \pm 2.2$ \\
\hline \multicolumn{9}{|c|}{ IB oily solution $(25 \mathrm{mg} \mathrm{IB} / \mathrm{kg})$} \\
\hline 13 & 37.36 & 2.07 & $0.01^{a}$ & 0.00 & $0.01^{a}$ & Not analysec & & \\
\hline 14 & 25,28 & 0.90 & $0.14^{a}$ & $0.10^{a}$ & 0.00 & & & \\
\hline 15 & 54,74 & 0.72 & $0.08^{a}$ & 0.00 & 0.00 & & & \\
\hline 16 & 10.01 & $0.04^{a}$ & 0.00 & 0.00 & 0.00 & & & \\
\hline 17 & 19.47 & $0.06^{a}$ & 0.00 & 0.00 & 0.00 & & & \\
\hline 18 & 38.41 & 0.33 & 0.00 & 0.00 & $0.03^{a}$ & & & \\
\hline Mean \pm SD & $30.88 \pm 15.91$ & $0.69 \pm 0.76$ & $0.04 \pm 0.06$ & $0.02 \pm 0.04$ & $0.01 \pm 0.01$ & & & $48.4 \pm 25.1$ \\
\hline
\end{tabular}

${ }^{a}$ Values below quantification limit

${ }^{r}$ Data rejected

1.50 while the $\mathrm{pH}$ was 6.10 . This did not change during sterilisation process.

\section{Pharmacokinetics of IB in Rabbits}

The IB plasma concentrations after administration of each formulation are shown in Table I and Fig. 3. Except for two cases (animals 2 and 8, Table I), the maximum concentration of IB in plasma was detected after approximately $1 \mathrm{~h}$, irrespective of the type of formulation. Average IB $\mathrm{C}_{\max }$ values for ibuprofen-OHB preparations were 1.3 and $1.0 \mu \mathrm{g} / \mathrm{ml}$ for the oily solution and emulsion, respectively. However, this difference was not found to be significant $(p<0.05)$. A higher concentration of IB in plasma $\left(\mathrm{C}_{\max } 48.5 \mu \mathrm{g} / \mathrm{ml}\right)$ was found after injection of IB compared with ibuprofen-OHB, despite administration of a lower dose of IB $(25 \mathrm{mg} / \mathrm{kg}$ versus $40 \mathrm{mg} / \mathrm{kg}$ in case of ibuprofen-OHB). Six hours after injection, detectable IB concentrations were only observable in plasma of three rabbits receiving free IB; however, these samples were below the limit of quantification. In contrast, following injection of ibuprofen-OHB formulations, detectable IB plasma concentrations (up to $1 \mu \mathrm{g} / \mathrm{ml}$ ) were found at the time points up to $24 \mathrm{~h}$. Four samples (Table I) were rejected from the analysis due to incoherence with the expected pharmacokinetic profile (rabbit 1;24 h) or with values measured at the same conditions for the other animals from the same group (rabbit 7). It should be
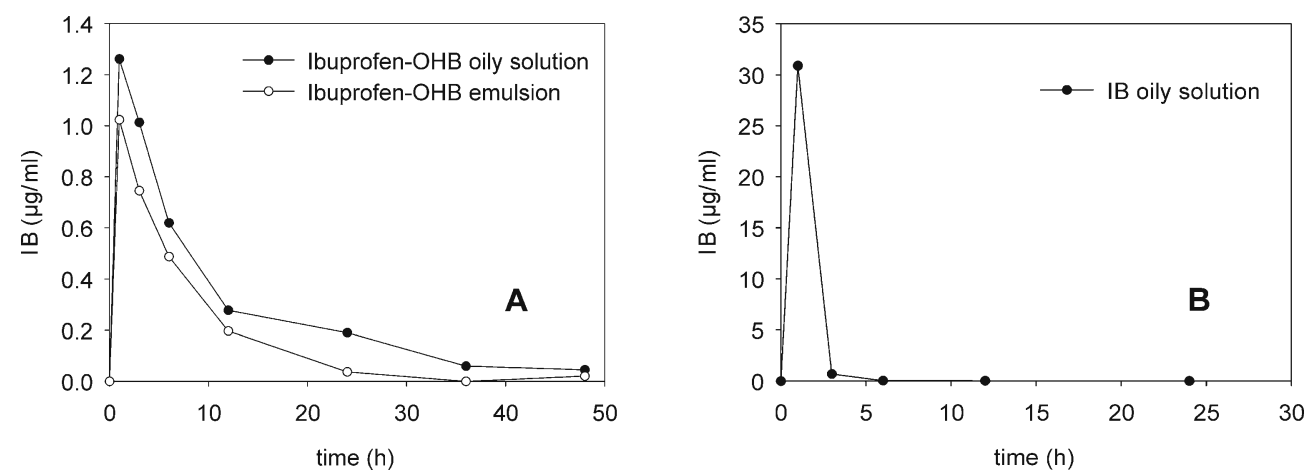

Fig. 3. Pharmacokinetic profiles of tested IM formulations: a Ibuprofen-OHB oily solution and emulsion (40 mg IB/ $\mathrm{kg})$, b IB oily solution $(25 \mathrm{mg} / \mathrm{kg}$ ). Values are means of at least five independent experiments $\left(p^{*}<0.05\right.$; Mann-Whitney rank sum test) 
stressed that the AUC values were calculated on a basis of all obtained results, also these below LOQ, so they should be treated rather as approximations (however, the precision calculated as a RSD between single injections did not exceed $15 \%$ also for $\mathrm{C}$ values below $0.2 \mu \mathrm{g} / \mathrm{ml}$ ). These values were still reported (Table I) due to the fact that their rejection did not significantly change the final AUC (e.g. the values for ibuprofen-OHB emulsion were 7.9 and $7.15 \mu \mathrm{g} / \mathrm{ml} \cdot \mathrm{h}$, respectively). Moreover, the precision was assessed as sufficient to demonstrate the time of drug release.

Although the difference between AUCs obtained for ibuprofen-OHB preparations $(13.2 \mu \mathrm{g} / \mathrm{ml} \cdot \mathrm{h}$ for the oily solution and $7.9 \mu \mathrm{g} / \mathrm{ml} \cdot \mathrm{h}$ for the emulsion) may indicate a superior bioavailability of the pro-drug administered as an oily solution, statistical analysis did not confirm significance $(p=0.129)$ between both preparations. Statistically significant difference, however, was shown $24 \mathrm{~h}$ after injection, when the concentration of IB $(0.19 \mu \mathrm{g} / \mathrm{ml}$, with results below LOQ $)$ following administration of the oily solution of ibuprofenOHB was higher than after injection of the emulsion (all results below LOQ).

A pronounced difference in bioavailability was demonstrated between IB and ibuprofen-OHB preparations. Despite a being nearly two times the lower dose of IB, AUC values calculated for IB oily solution $(48.4 \mu \mathrm{g} / \mathrm{ml} \cdot \mathrm{h})$ exceeded the AUC values for both ibuprofen-OHB preparations by four to six times. Consequently, the relative bioavailability of IB bounded to OHB compared with the IB preparation was relatively low $(17.0 \%$ for the oily solution and $10.0 \%$ for the emulsion).

\section{DISCUSSION}

A HPLC method was developed for the analysis of IB in rabbit plasma which proved to be reliable, precise and accurate. An internal standard (IND) chosen on a basis of previous findings (12) allowed for appropriate peak separation. Although some authors obtained better validation parameters, especially, with regards to the recovery of the analysed substance $(13,14)$, others reported values similar to our findings (15).

An initial increase of IB plasma levels following ibuprofen-OHB administration with relatively early $T_{\max }$ values identical to the non-conjugated IB preparation can be attributed to free IB present in the ibuprofen-OHB formulation. Administration of $25 \mathrm{mg} \mathrm{IB} / \mathrm{kg}$ as an oily solution resulted in a $\mathrm{C}_{\max }$ of approx $30 \mu \mathrm{g} / \mathrm{ml}$. Since the content of the non-conjugated drug in ibuprofen-OHB was $\sim 2.5 \%$ (9), the animals receiving $120 \mathrm{mg} / \mathrm{kg}$ of ibuprofen-OHB (40 mg of IB), received a dose of $\sim 1.0 \mathrm{mg} / \mathrm{kg}$ of non-conjugated drug. Hence, the expected $\mathrm{C}_{\max }$ of $1.2 \mu \mathrm{g} / \mathrm{ml}$ was confirmed in the experiment.

Hydrolytic breakdown of ibuprofen-OHB in vivo was confirmed by the presence of IB in the samples collected 6 , 12 and $24 \mathrm{~h}$ after administration, while in rabbits receiving free IB, the drug could no longer be detected. The very slow rate of hydrolysis is also consistent with results obtained previously in vitro, revealing after $72 \mathrm{~h}$, only $15 \%$ of non-conjugated IB in $\mathrm{pH} 6.8$ simulated intestinal fluid, and no significant increase in IB concentration in different phosphate-buffered solutions at a $\mathrm{pH}$ range 7.0 8.0 (9). It can thus be deduced that, due to low hydrolytic rate, almost all of the substance remains in the muscle tissue.

Ibuprofen-OHB allowed for sustained release of the active compound, although the slow hydrolysis of the conjugate resulted in low plasma concentrations. This phenomenon coincides with the published results of several other groups $(16,17)$ suggesting that the hydrolysis rate of drug conjugates depends on the nature of chemical linkage between the active molecule and the conjugated substance. Faster hydrolysis rate would require incorporation of an appropriate spacer group with hydrolytically unstable bonds between the IB and OHB moieties, which has been demonstrated to accelerate hydrolysis and allows for detection of considerably higher drug levels in blood (17). The observed hydrolysis profile makes the oligo(3-hydroxybutyric acid) conjugates particularly interesting for monocarboxylic acid drugs with considerable pharmacological activity at low doses.

\section{CONCLUSION}

No significant differences in pharmacokinetic characteristics between ibuprofen-OHB emulsion and oily solution have been demonstrated. In comparison to nonconjugated model drug, the conjugated form allowed for prolonged release of the active substance. It can be concluded that, due to reported lack of toxicity (5) and sustained release characteristics, drug conjugates with oligo (3-hydroxybutyric acid) are promising candidates for parenteral drug carriers.

\section{ACKNOWLEDGEMENTS}

This work was supported by a Galenos Fellowship in the Framework of the EU Project "Towards a European $\mathrm{PhD}$ in Advanced Drug Delivery", Marie Curie Contract MEST-CT2004-4049922. The authors wish to thank Ms. Anna Kowalczyk and Ms. Iwona Kalinowska for their excellent technical assistance during the experiments.

Open Access This article is distributed under the terms of the Creative Commons Attribution Noncommercial License which permits any noncommercial use, distribution, and reproduction in any medium, provided the original author(s) and source are credited.

\section{REFERENCES}

1. Pasut G, Veronese FM. Polymer-drug conjugation, recent achievements and general strategies. Prog Polym Sci. 2007;32:93361.

2. Hamidi M, Azadi A, Rafiei P. Pharmacokinetic consequences of pegylation. Drug Deliv. 2006;13:399-409.

3. Duncan R, Vicent MJ, Greco F, Nicholson RI. Polymer-drug conjugates: towards a novel approach for the treatment of endrocine-related cancer. Endocr Relat Cancer. 2005;12:18999. 
4. Khandare J, Minko T. Polymer-drug conjugates: progress in polymeric prodrugs. Prog Polym Sci. 2006;31:359-97.

5. Piddubnyak V, Kurcok P, Matuszowicz A, Glowala M, FiszerKierzkowska A, Jedlinski Z, et al. Oligo-3-hydroxybutyrates as potential carriers for drug delivery. Biomaterials. 2004;25:5271-9.

6. Zinn M, Witholt B, Egli T. Occurrence, synthesis and medical application of bacterial polyhydroxyalkanoate. Adv Drug Deliv Rev. 2001;53:5-21.

7. Jedlinski Z, Juzwa M, Zawidlak-Wegrzynska B, Kaczmarczyk B, Bosek I, Wanic A. Novel esters of nonsteroidal anti-inflammatory drugs and methods of their preparation. Polish Patent PL 196384, 2007.

8. Juzwa M, Rusin A, Zawidlak-Wegrzynska B, Krawczyk Z, Obara I, Jedlinski Z. Oligo(3-hydroxybutanoate) conjugates with acetylsalicylic acid and their antitumour activity. Eur $\mathrm{J}$ Med Chem. 2008;43:1785-90.

9. Stasiak P, Ehrhardt C, Juzwa M, Sznitowska M. Characterisation of a novel conjugate of ibuprofen with 3-hydroxybutyric acid oligomers. J Pharm Pharmacol. 2009;61:1119-24.

10. Zawidlak-Wegrzynska B, Kawalec M, Bosek I, Luczyk-Juzwa M, Adamus G, Rusin A, et al. Synthesis and antiproliferative properties of ibuprofen-oligo(3-hydroxybutyrate) conjugates. Eur J Med Chem. 2010;45:1833-42.
11. http://www.phenomenex.com/AppManager/Files/CN007_Extraction \%20of\%20Ibuprofen \%20from \%20Plasma \% 20using \%20strata-X.pdf. Accessed 13 August 2009.

12. Sochor J, Klimes J, Sedlacek J, Zahradnícek M. Determination of ibuprofen in erythrocytes and plasma by high performance liquid chromatography. J Pharm Biomed Anal. 1995;13:899-903.

13. Farrar H, Letzig L, Gill M. Validation of a liquid chromatographic method for the determination of ibuprofen in human plasma. J Chromatogr B. 2002;780:341-8.

14. Bonato PS, Del Lama MP, de Carvalho R. Enantioselective determination of ibuprofen in plasma by high-performance liquid chromatography-electrospray mass spectrometry. J Chromatogr B. 2003;796:413-20.

15. Gallardo A, Parejo C, San Roman J. NSAIDs bound to methacrylic carriers: microstructural characterization and in vitro release analysis. J Control Release. 2001;71:127-40.

16. Zhao X, Tao X, Wei D, Song Q. Pharmacological activity and hydrolysis behavior of novel ibuprofen glucopyranoside conjugates. Eur J Med Chem. 2006;41:1352-8.

17. Babazadeh M. Synthesis and study of controlled release of ibuprofen from the new acrylic type polymers. Int J Pharm. 2006;316:68-73. 